\title{
Appropriation et stratégies d'intégration des médias sociaux par les professionnels de la communication
}

Francine Charest, Anne-Marie Gauthier et François Grenon

\section{CpenEdition}

\section{Journals}

Édition électronique

URL : http://journals.openedition.org/communicationorganisation/4259

DOI : 10.4000/communicationorganisation.4259

ISSN : 1775-3546

Éditeur

Presses universitaires de Bordeaux

Édition imprimée

Date de publication : 1 juin 2013

Pagination : 269-280

ISBN : 978-2-86781-883-7

ISSN : $1168-5549$

\section{Référence électronique}

Francine Charest, Anne-Marie Gauthier et François Grenon, « Appropriation et stratégies d'intégration des médias sociaux par les professionnels de la communication », Communication et organisation [En ligne], 43 | 2013, mis en ligne le 01 juin 2015, consulté le 10 décembre 2020. URL : http:// journals.openedition.org/communicationorganisation/4259; DOI : https://doi.org/10.4000/ communicationorganisation.4259 


\title{
Appropriation et stratégies d'intégration des médias sociaux par les professionnels de la communication
}

\author{
Francine Charest ${ }^{1}$, Anne-Marie Gouthier ${ }^{2}$, Fronçois Grenon ${ }^{3}$
}

\author{
LinkedIn (2003), Facebook (2004), YouTube (2005), Twitter (2006)
} et les autres changent la donne dans les pratiques des professionnels de la communication. Tout comme pour le Web, devenu accessible au grand public en 1994 (Castells, 2002) et considéré, à peine une décennie plus tard, comme l'un des plus puissants outils planétaires de communication (Charest et Bédard, 2009), la question n'est plus de se demander si les organisations ${ }^{4}$ doivent intégrer ces réseaux à leurs stratégies de communication, mais plutôt comment ? (Tapscott et Williams, 2006). Les médias ${ }^{5}$ sociaux, ces plates-formes collaboratives et évolutives, obligent les organisations à d'importantes mutations dans de très courts laps de temps. Notamment, parce qu'ils n'ont pas été créés à des fins de communication de masse, mais bien de réseautage. Ces changements confèrent aux relationnistes et aux communicateurs le devoir d'observer assidûment " les pratiques et les usages qui s'y développent » (Stenger et Coutant, $2010: 221$ ), et ainsi faire

\footnotetext{
1 Francine Charest est professeure adjointe au département d'information et de communication (DIC) de l'Université Laval, à Québec au Canada. Elle a créé le centre de recherche l'Observatoire des médias sociaux en relations publiques de l'Université Laval. Elle en assure la direction en collaboration avec des professeurs du département, majoritairement regroupés dans la concentration des relations publiques. Elle est habilitée à diriger des travaux de recherche et de réflexion dans le domaine de la communication et des pratiques professionnelles dans les médias sociaux ; francine.charest@com.ulaval.ca

2 Anne-Marie Gauthier est finissante à la maîtrise en communication publique, profil relations publiques, à l'Université Laval. Elle a rédigé un essai sur l'appropriation des médias sociaux par les professionnels en relations publiques. Membre du groupe d'experts-conseil de l'Observatoire des médias sociaux en relations publiques, elle travaille également comme chargée de projet et responsable des communications Web dans un organisme gouvernemental québécois ; annemariegauthier@hotmail.com

3 François Grenon cumule les tâches de chargé d'enseignement au Département d'information et de communication de l'Université Laval et de stratège/consultant en communication, relations publiques. Il est également finissant à la maîtrise en communication publique, profil relations publiques de l'Université Laval. Intéressé par l'avènement des médias sociaux et leur impact pour les organisations publiques et privées, il est particulièrement sensible aux stratégies qui favorisent leur intégration; francois.grenon@sivis.ca

4 Le terme organisation dans ce texte regroupe autant les entreprises, les organisations privées ou publiques, que les organismes à structure associative.

5 Les auteurs voient davantage les réseaux sociaux comme de véritables médias.
} 
évoluer les processus stratégiques en fonction de l'appropriation qu'en font les utilisateurs.

Certaines règles s'appliquent. Les communications corporatives doivent s'inscrire dans la logique des médias sociaux faite d'interactions, de relations et de conversations pour ainsi délaisser le mode conventionnel de communication organisationnelle : la diffusion d'information. Les stratégies développées doivent alors tenir compte des valeurs inhérentes à ces médias, proches des cinq indicateurs de la théorie des relations de Ledingham et Bruning (1998) : la fiabilité, la transparence, l'implication, l'investissement et l'engagement à long terme. En outre, les stratégies prescrites doivent prendre en compte les tactiques inventées au quotidien (de Certeau, 1990) par les milliers d'usagers lors de leur appropriation des nouveaux outils.

Le présent article recense les réflexions stratégiques d'auteurs et spécialistes du Web et des médias sociaux. À la lumière de lectures, d'expériences et d'observations empiriques, un processus stratégique d'appropriation et d'intégration des médias sociaux en dix étapes est présenté, ainsi que des recommandations et écueils.

\section{Processus d'intégration en dix étapes}

\section{$1^{\text {ère }}$ étape : veiller et observer}

Chaque organisation devrait dresser son portrait web afin de repérer les zones d'influence liées à cette dernière. Pour ce faire, un exercice d'écoute et d'analyse de ce qui se dit et se fait sur le Web et dans les médias sociaux devrait constituer un corpus de départ, ou veille préliminaire. Pour définir par la suite la stratégie à adopter, il est primordial de connaître cette empreinte numérique.

Comme le martèle Mitch Joël : « Ce n'est pas vous qui définissez votre marque. C'est Google » (2010 : 24). La veille préliminaire devrait donc inclure les traces laissées en ligne par les internautes (positives ou négatives), les commentaires et les billets de blogues, le comportement des concurrents ou des opposants, etc. Observer ce que font les organisations de même nature permet de s'en inspirer ou de s'en démarquer. "La présence des concurrents sur le Web est une sorte de laboratoire à ciel ouvert qui permet d'en analyser les actions de communication» (Dupin, $2010: 40$ ).

Lidentification des groupes ou des comptes dans les médias sociaux, non officiels, mais en lien direct avec l'organisation permettra de repérer les " usages détournés ${ }^{6}:$ "la présence de ces comptes factices peut venir parasiter toute forme de communication » (Dupin, 2010: 37). Cependant, le résultat peut aussi être positif, si le compte a été créé de bonne foi et qu'il rallie

6 Termes consacrés par Michel de Certeau (1990). 
déjà des adeptes (fans) de l'organisation ou de la marque. Il est alors possible de se réapproprier le compte en communiquant avec les initiateurs.

Le résultat de cette veille préliminaire pourrait en surprendre plusieurs comme le soulignent Blanc et Seraiocco (2010 : 43) : «Cette étape pourrait aussi vous convaincre d'agir, car si, par souci de ne pas perdre le contrôle du message, vous vous abstenez encore de tremper dans le Web social, j'ai des petites nouvelles pour vous : vous y êtes probablement déjà ! ». Il s'avère donc crucial d'effectuer une veille préliminaire rigoureuse sur le Web et les médias sociaux. Pour y arriver, on peut utiliser un amalgame d'outils gratuits dont Google Alerts ${ }^{7}$ (pour les contenus indexés par Google), Hootsuite ${ }^{8}$ et Social Mention ${ }^{9}$ (recherche par mots-clés sur le Web et dans les médias sociaux), Tweetdeck ${ }^{10}$ (recherche par mots-clés en temps réel sur Twitter) ou encore l'abonnement à des fils RSS. Pour ceux qui en ont les moyens financiers, Radian6 ${ }^{11}$ ou Voxco ${ }^{12}$ (tableau de bord analysant le Web social de façon qualitative et quantitative) pourraient être envisagés. Dupin rappelle toutefois qu'une veille réalisée par une personne physique « est probablement la plus pertinente, car elle prend en considération un environnement auquel les moteurs de recherche n'ont pas forcément accès " (2010:34). Ce travail de veille prend du temps, et il n'est pas rare d'y consacrer quelques semaines. La phase d'observation représente également une étape primordiale pour Marc Snyder (cité dans Malaison, 2007) : « trouver des blogues et des médias sociaux qui parlent de notre organisation ou de notre industrie. Ensuite, il faut " écouter », $[\ldots]$ et suivre ainsi les conversations. Ce n'est que par la suite qu'il faut « commenter », puis, ultimement, "participer » en intégrant la blogosphère et autres médias sociaux (Malaison, 2007 : 108-109).

Enfin, bien que cette étape de veille préliminaire soit ici identifiée en premier lieu, elle doit se poursuivre tout au long de la mise en place et de la réalisation de la stratégie média social, pour devenir permanente par la suite et demeurer l'une des priorités de toute organisation. Celle-ci doit répondre rapidement aux commentaires qui lui sont destinés, et cette veille permet d'anticiper les situations de communication sensible, d'observer la concurrence et d'être à l'affût des tendances et des nouveautés du Web social. «Les blogues et les médias sociaux peuvent être à la fois un système de détection des tendances et des enjeux à venir et à la fois des outils pour que votre entreprise puisse être un leader dans son domaine " (Snyder dans Malaison, 2007 : 103). De plus, dresser le portrait de la situation Web actuelle de l'organisation « sera utile par la suite pour mesurer votre progression » (Blanc et Seraiocco, 2010 : 34).

7 http://www.google.ca/alerts

8 http://hootsuite.com/

9 http://www.socialmention.com/

10 Tweetdeck.com

11 http://www.radian6.com/

12 http://www.voxco.com 


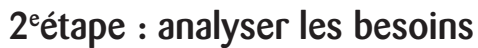

L'analyse des besoins internes et externes est primordiale afin d'assurer une intégration conséquente et réussie. Elle permettra par exemple de dégager qu'une organisation souhaite y présenter ses produits tandis qu'une autre préfèrera y gérer ses relations avec la clientèle alors que la plupart souhaiteront simplement exercer une veille active et engager la conversation avec les publics intéressés. Cela dit, l'analyse des besoins doit également, et principalement, être tournée vers les utilisateurs externes afin de connaître leurs attentes spécifiques aux médias sociaux. Charest et Bédard (2009) inspirés par Nielsen, avancent que des erreurs fondamentales se produisent dans les stratégies des concepteurs de nouveaux médias « dont le modèle d'affaires est d'abord limité à un usage de diffusion qui reflète les besoins des entreprises plutôt que ceux des clients » (Ibid. : 84).

\section{$3^{\text {e }}$ étape : identifier les publics ou clientèles et établir les objectifs}

Une fois les besoins identifiés, l'organisation choisira de s'attaquer dans un premier temps aux plus urgents ou aux plus faciles à répondre. Les priorités établies, il sera aisé d'identifier les publics (ou clientèles) visés, de même que les objectifs de présence. Également, comme dans toute stratégie de communication, l'entreprise doit définir ses objectifs de communication. Cette réflexion peut s'articuler autour des « $5 \mathrm{~W}$ » élaborés par Lasswell dès 1948, appliqués en relations publiques et en journalisme, et repris par Dupin : pourquoi ? Qui ? Quoi ? Comment et par qui ? Combien ? Où ? L'auteur propose de surcroît d'utiliser la méthodologie SMART pour déterminer les objectifs : spécifique, mesurable, atteignable, réaliste et temporel (2010:65). L'auteur met toutefois en garde les lecteurs face aux objectifs mercantiles des stratégies : «Attention, les médias sociaux reposent d'abord et avant toute chose sur l'engagement, la cocréation et sur la valorisation d'une image. La vente et l'apport de trafic sont dans ce sens de mauvais objectifs» (Ibid. : 65).

\section{$4^{\mathrm{e}}$ étape : choisir les outils et créer les profils}

Lorsque les objectifs et les publics (ou clientèles) sont identifiés, les médias à privilégier s’imposeront naturellement.

En outre, l'organisation doit se limiter et choisir les canaux répondant réellement à sa stratégie, et aux profils de ses publics, plutôt que d'adopter ceux qui s'inscrivent dans un "effet de mode " (Morin, 2010). Aussi, il est important de sécuriser le nom de l'organisation sur les différentes plateformes sociales, de façon à éviter le cybersquattage (Blanc et Seraiocco, 2010 : 34). Par prudence, la réservation des noms et adresses URL se fera en tout début de processus en choisissant ceux qui seront facilement repérables par les internautes et les moteurs de recherche. 


\section{$5^{\mathrm{e}}$ étape : prévoir des contenus pour chaque média}

Une stratégie média social repose sur une dualité : savoir s'adapter aux contenus des internautes et être capable d'enrichir la conversation. Avant d'intégrer un média en particulier, l'organisation définira au préalable des contenus pertinents à utiliser : textes, archives, capsules vidéos, photos, dossiers d'intérêt, événements, etc. Selon Dupin, une organisation qui ne produit pas suffisamment de contenu pour sa communauté la rend inerte, ce qui entraîne un désintérêt et une diminution de l'interaction $(2010$ : 37). Voilà pourquoi cette étape est si importante. "Il est majeur de travailler le contenu des messages qui alimenteront la communauté sur le réseau social. Ce contenu doit apporter systématiquement de la valeur ajoutée, des recommandations, des suggestions, les réponses aux besoins exprimés par les membres » (Balagué et Fayon, $2010: 83$ ). «Dès le départ, apportez une attention particulière aux contenus que vous entendez diffuser. Ils constituent la pierre angulaire de votre image dans le réseau » (Morin, 2010 : 51).

Il est donc recommandé de se créer un calendrier éditorial et de se donner le droit d'être spontané pour conserver le rythme et l'intérêt selon l'actualité et les conversations en cours : "N'hésitez pas à remettre certains sujets pour commenter une nouvelle de dernière minute concernant votre marché ", conseille Mitch Joël (2010 : 119).

\section{$6^{e}$ étape : adopter des outils de gestion}

Cette phase constitue l'une des plus névralgiques ; toutefois, elle est trop souvent négligée par les organisations. Les dirigeants ont le devoir d'encadrer les pratiques de leurs employés dans les médias sociaux et de pourvoir l'organisation d'outils de gestion performants. Comme le souligne Stenger, «On ne peut pas ne pas prescrire» des contenus sur les réseaux socionumériques (2011: 128). L'organisation doit donc s'interroger sur les façons de le faire. Par exemple, les employés ont-ils accès à ces plates-formes sur leurs heures de travail ? Qui sera responsable de la gestion des profils corporatifs? Comment répond-on aux commentaires ? Quel contenu diffuse-t-on ?

Afin de mieux encadrer les usages, nous proposons de créer une charte comprenant certains items incontournables, lesquels sont parfois le simple prolongement de politiques déjà adoptées par les organisations :

Le guide d'utilisation des médias sociaux permet de rappeler les objectifs de présence et les plateformes choisies. On y identifie les gestionnaires et les considérations importantes à appliquer lors des échanges avec les internautes, notamment le lexique d'usage et le niveau de langage approprié. Cette "bible des médias sociaux » peut inclure également une charte d'utilisation personnelle des médias sociaux, destinée aux employés de l'organisation. Bien que la majorité des employés, dont les plus jeunes (les digital natives), soient relativement à l'aise avec les règles tacites des médias sociaux, il s'avère fort utile de rappeler certains concepts entourant la confidentialité et la pérennité 
des traces laissées sur le Web. On oublie trop souvent la mince frontière qui existe entre le domaine privé et le domaine public, propre aux médias sociaux.

La politique éditoriale permet d'établir clairement les sujets d'intérêt qui seront traités dans les médias sociaux, tout comme les sujets qui seront évités.

La politique de gestion des commentaires illustre la manière dont l'organisation réagit ou non, aux commentaires laissés par les internautes. Dans leurs livres, Dupin et aussi Blanc et Seraiocco, reprennent le célèbre schéma de gestion des commentaires de l'US Air Force pour illustrer adéquatement la manière de répondre et d'appréhender les commentaires négatifs sur la Toile (Dupin, 2010 : 28 ; Blanc et Seraiocco, 2010 : 70). Ce processus se fait en trois étapes : la découverte d'un commentaire, l'évaluation (typologie du commentaire) et la réponse (contenu pertinent et transparent). Dans ce processus de réponse, cinq considérations sont mises de l'avant, soit la transparence, les références, le temps, le ton et l'influence.

L'Observatoire des médias sociaux en relations publiques de l'Université $\mathrm{Lava}^{13}$ a également développé une expertise en planification de gestion des commentaires. Un schéma indique au gestionnaire chacune des actions à prendre ainsi que le délai à respecter, selon la nature du commentaire. De plus, un système visuel de couleurs permet d'identifier rapidement des propos concernant une situation de communication sensible, devant être rapportée à la direction des relations publiques.

L'adoption d'outils de gestion permet ainsi la diffusion d'un contenu cohérent, tout en conférant une autonomie et une efficacité aux gestionnaires des médias sociaux. Mais encore faut-il que ces outils soient connus de tous les employés. Enfin, l'élaboration des différents outils de gestion devrait se faire dans l'esprit de collaboration propre aux médias sociaux donc, avec les employés. Ainsi, leur appropriation sera facilitée lors de leur adoption officielle par les dirigeants.

\section{$7^{e}$ étape : former et mobiliser les ressources}

Bien que l'adoption de normes et de politiques soit nécessaire pour encadrer les pratiques, «il ne s'agit pas uniquement de fixer des règles : il est important de former son personnel à la pratique et aux enjeux des médias sociaux et aux nouvelles logiques clients " (Dupin, 2010 : 26). Un nouveau métier de communication voit le jour pour répondre à cette demande : le gestionnaire de communauté. Ce rôle-clé « regroupe différentes facettes : représenter la communauté, évangéliser le marché, communiquer, écouter et analyser les billets publiés » (Balagué et Fayon, 2010 : 99).

Au-delà du gestionnaire de communauté, l'organisation doit identifier des ressources compétentes pour agir parfois à titre d'expert et apporter une valeur ajoutée. Baer et Naslund, dans leur récent ouvrage The Now Revolution

13 http://www.com.ulaval.ca/recherche/groupes-de-recherche/observatoire-des-medias-sociaux-en-relationspubliques/ 
(2011), s'accordent sur le fait que les stratégies déployées pour intégrer les médias sociaux doivent se refléter à l'intérieur de l'organisation, au sein d'une culture forte, partagée et évolutive, chaque membre devant être considéré comme un porte-parole potentiel.

Toujours en termes de cohérence, l'esprit collaboratif des médias sociaux doit se refléter prioritairement dans les actions à l'interne. «Pour éviter de vous orienter vers des changements qui ne correspondent pas aux véritables besoins de votre organisation, impliquez vos employés clés dès le départ. Faites-leur confiance et consultez-les à chacune des étapes »(Morin, 2010 : 35). Enfin, l'une des raisons souvent évoquées pour ne pas intégrer les médias sociaux est le manque de temps et de ressources. Pour éviter la surcharge, il est alors recommandé de former un groupe d'administrateurs qui se partageront les tâches.

\section{$8^{e}$ étape : engager la conversation (interagir, échanger et partager)}

Ce n'est donc qu'après avoir complété les sept étapes précédentes que l'organisation est fin prête à interagir dans les médias sociaux.

Grâce aux plateformes collaboratives et aux médias sociaux, "les réseaux socionumériques confèrent la possibilité à l'usager de participer de manière active à la production de contenus médiatiques propres " (Proulx et Choon, 2011 : 105). Morin ajoute " la clé du succès d'une présence dans les sites de réseautage social réside dans la qualité des interventions des utilisateurs » (2010 : 51).

Ces interventions doivent être alimentées par l'organisation elle-même, dans le respect de la logique des médias sociaux. «L'enjeu pour l'entreprise est d'apparaître dans le flux continu des échanges [...] et de faire partie des conversations en ligne ", proposait le PDG de Facebook, Zuckergerg en 2007, rappelle Thomas Stenger (2011: 130).

L'organisation conservera ainsi l'intérêt et mettra l'internaute au centre du processus communicationnel, alors que " le monologue des ex-influenceurs est devenu le dialogue des masses ; c'est ainsi que l'ancienne façon de communiquer vers les publics doit céder sa place à communiquer avec les publics " (Snyder dans Malaison, 2007 : 104). Ces notions d'interaction, d'échange et de partage rejoignent les fondements de certaines théories de relations publiques, dont la gestion des relations (Ledingham et Bruning, 1998) présentée plus tôt, et le modèle de communication bidirectionnelle symétrique (Grunig, 1984). Ce dernier modèle concède un pouvoir réel aux groupes communicants quant aux décisions organisationnelles les concernant.

En somme, pour créer de vrais dialogues et des échanges constructifs avec les publics, le communicateur doit s'amuser. Évidemment, l'entretien et l'animation des conversations constituent le travail au quotidien de ce dernier, mais les internautes doivent tout de même sentir sa passion et son enthousiasme. "There are so many channels out there. Find the ones you really 
enjoy and create the type of content that gives you the most pleasure. Find your muse $»^{14}$.

Désormais, il s'agit de diffuser des contenus pertinents, adaptés aux publics ou clientèles selon les différents canaux, et d'offrir une valeur ajoutée. «Vous devez donc emprunter ce que j'appelle la longue route : consacrez une grande partie de votre temps et de vos efforts à la création d'un contenu qui interpellera les consommateurs ciblés et qui les conviera à une expérience unique " (Joël, 2010 : 70). Comme le stipule Morin, « le succès d'une démarche dans les réseaux sociaux dépend essentiellement de la qualité des contributions et des contacts qu'on procure à la communauté. Sans cet apport, le lien de confiance n'existe pas, et le réseautage social s'avère vain et inefficace» $(2010: 45)$.

\section{9 étape : au besoin, créer une communauté d'intérêts}

Il faut savoir reconnaître les influenceurs et les alliés potentiels, ces leaders d'opinion qu'il sera avantageux de compter parmi vos réseaux afin qu'ils puissent relayer les communications. "Il est important de transformer sa communauté pour qu'elle ne soit plus une simple audience, mais bien un relai »(Dupin, 2010: 143).

Le développement de la communauté doit se baser sur une stratégie à long terme. A cet égard, dans un article paru dans la revue Réseaux, Boullier souligne que « la fidélisation est devenue un vecteur essentiel pour assurer une entrée privilégiée dans l'univers de la personne et donc capter son attention » (2009: 235).

Mitch Joël accorde une grande importance aux stratégies pour bâtir une communauté et il avance que « la vie d'une communauté ne se limite pas aux activités ayant lieu dans les sites que vous gérez. Une communauté virtuelle saine fréquente plusieurs sites. Explorez les divers moyens d'être présent et connecté, chez vous et ailleurs» (2010:77). Toutefois, il ne faut pas confondre intérêt et implication, si plusieurs internautes sont rapides à vous aimer, peu prendront votre défense le temps venu.

\section{$10^{\mathrm{e}}$ étape : évaluer et ajuster, établir une veille permanente}

Une fois la stratégie d'intégration déployée, il importe de mesurer l'atteinte ou non des objectifs préétablis. Par contre, « Le calcul du RoI (return on investment) des actions sur les réseaux sociaux reste à ce jour un exercice difficile, car il suppose de transformer en données quantitatives des interactions ou des conversations entre les individus " (Balagué et Fayon, 2010 : 166). Les outils qui permettent de mesurer l'atteinte des objectifs s'appuieront sur des indicateurs qui se nourriront non seulement des opinions et des perceptions des consommateurs recueillies avec des techniques de mesure plus traditionnelles, mais également de leurs comportements et de 
leurs conversations mesurées en temps réel sur le web (Ruette-Guyot et Leclerc : 140). À cet effet, les outils de mesure et de veille stratégique dont Google Analytics ${ }^{15}$, les statistiques des pages Facebook, Radian6, Hootsuite, Bit. ly ${ }^{16}$, etc., sont ici extrêmement utiles.

L'exercice doit, cependant, se faire tout au long du processus d'échange avec les internautes. Le rôle de gestionnaire de communauté et des communicateurs consiste donc à mesurer constamment l'impact des actions de façon à identifier les influenceurs, les contenus et les médias sociaux qui suscitent le plus les conversations. Ainsi, le tir peut être réajusté rapidement, en fonction des intérêts de la communauté, des tendances et de l'actualité.

Il importe également de poursuivre sur la base de la veille préliminaire, une veille permanente, quotidienne et en temps réel. Bien que cela paraisse lourd, une fois les mots-clés identifiés, une petite organisation ne prendra que quelques heures par jour à observer (et au besoin réagir) ce qui se dit sur le Web et dans les médias sociaux.

\section{Conclusion}

À partir de la littérature et d'observations, il nous a été possible de proposer une démarche pour réussir l'appropriation et l'intégration des médias sociaux dans une stratégie de communication corporative. Il faut néanmoins se rappeler que ces étapes ne représentent guère une recette miracle et que chaque entreprise a le devoir de s'inscrire dans la logique des médias sociaux en adhérant à ses valeurs intrinsèques pour ensuite analyser son environnement afin de trouver sa voie. De plus, compte tenu de l'évolution effervescente du Web collaboratif, de nombreuses mutations sont à prévoir dans l'univers des médias sociaux. Il importe donc aux professionnels de la communication et aux chercheurs de demeurer à l'affût des tendances. En outre, les stratégies prescrites doivent prendre en compte les façons de faire des internautes dans leur appropriation des nouveaux outils de communication, que ces usages soient prévus ou « détournés " par certains détracteurs.

Enfin, bien que nous nous retrouvions au sein d'une nouvelle communication interactive, l'improvisation n'a guère sa place. Ce qui est intemporel dans cette réflexion, tout comme dans toute action de communication, est qu'il est impératif de réfléchir avant d'agir.

Recommandations et écueils :

Fiabilité, transparence, implication, investissement et engagement à long terme, rejoignant ainsi les indicateurs de la théorie des relations de Ledingham et Bruning (1998) inscrits dans la logique des valeurs des médias sociaux, telles sont les recommandations qui émanent de nos observations et de nos lectures.

Certains écueils sont également à considérer lors de l'appropriation et de l'intégration des médias sociaux par les professionnels des relations publiques.

15 http://www.google.com/analytics/

16 Cet outil permet de générer des short URL et offre de bonnes possibilités de mesure : https://bitly.com/ 
Parler uniquement de soi, ne pas se préparer, ne pas avoir de gestionnaire de communauté et de relève, se penser meilleur que le voisin, utiliser de façon similaire toutes ses plateformes et utiliser le mauvais outil pour la clientèle à rejoindre... voilà quelques obstacles qu'il vous sera possible de contourner en adoptant une stratégie média social fondée sur le paradigme des interactions, la prise en compte des usages prescrits et effectifs, le respect de l'environnement de l'organisation et enfin, ce processus en dix étapes.

\section{BIBLIOGRAPHIE}

BAER J., NASLUND A., The Now Revolution, 7 Shifts to Make Your Business Faster, Smarter and More Social, Hoboken, John Wiley \& Sons, 2011.

BAER, J., Blogue Convice and Convert, "Why Social Succes Is Not Accident », [En ligne], http://www.convinceandconvert.com/social-media-strategy/why-social-successis-no-accident/ (Consulté le 28 juillet 2011).

BALAGUÉ C., FAYON D., Facebook, Twitter et les autres... Intégrer les réseaux sociaux dans une stratégie d'entreprise,Paris, Éditions Pearson, 2010.

BLANC M., SERAIOCCO N., Les médias sociaux 101, le réseau mondial des beauxfrères et des belles-sceurs, Montréal, Les Éditions Logiques, 2010.

BOULLIER D., "Les industries de l'attention : fidélisation, alerte ou immersion », Réseaux, 154, 2009, p. 231-246.

CASTELLS M., La Galaxie Internet, Éditions Fayard, Paris, 2002.

CERTEAU de M., L'Invention du quotidien,Tome 1 : Arts de faire, Paris, Gallimard, 1990.

CHAREST F., BÉDARD F., Les racines communicationnelles du Web, Québec, Éditions Presses de l'Université du Québec, 2009.

DUPIN A., Communiquer sur les réseaux sociaux, Paris, Éditions Flyp, 2010.

JOËL M., Six pixels de séparation. Le nouveau marketing à l'ère des médias sociaux, Montréal, Les Éditions Transcontinental, 2010.

JOËL M., Blogue Six Pixels of Separation, «How to Build Your Digital Footprint in 8 Easy Steps », [En ligne], http://www.twistimage.com/blog/archives/how-to-build-yourdigital-footprint-in-8-easy-steps/ (Consulté le 28 juillet 2011).

LEDINGHAM J., BRUNING S., « Relationship management in public relations: dimensions of an organization-public relationship », Public Relations Review, 24/1, 1998, p. 55-65.

MALAISON C., Pourquoi bloguer dans un contexte d'affaires, Montréal, Les Éditions Isabelle Quentin, 2007.

MORIN R., Comment entreprendre un virage 2.0., Montréal, Éditions Transcontinental, 2010.

PROULX S., KWOK CHOON M. J., «L'usage des réseaux socionumériques : une intériorisation douce et progressive du contrôle social ", Hermès, Ces réseaux numériques dits sociaux, 59, 2011, p. 105-111. 
RUETTE-GUYOT E., LECLERC S., Web 2.0 : la Communication "Iter-@ctive », Paris, Éditions Économica, 2009.

SERAIOCCO N., Blogue Chez Nadia, "Médias sociaux et relations publiques : la communication scénarisée », [En ligne], http://www.cheznadia.com/archives/2010/10/ medias-sociaux-et-relations-publiques-la-communication-scenarisee.html (Consulté le 15 juillet 2011).

SOCIAL MEDIA GOVERNANCE, Policy Database, [En ligne], http:// socialmediagovernance.com/policies.php (Consulté le 27 juillet 2011).

STENGER T., COUTANT A., "Les réseaux sociaux numériques : des discours de promotion à la définition d'un objet et d'une méthodologie de recherche ", Hermès, Journal of Language and Communication Studies, 44, 2010, p. 209-228.

STENGER T., "La prescription de l'action collective », Hermès, Ces réseaux numériques dits sociaux, 59, 2011, p. 127-133.

TAPSCOTT D., WILLIAMS A., Wikinomics, Wikipédia, Linux, YouTube... How Mass Collaboration Changes Everything, Pearson, 2006.

Résumé : L'appropriation des médias sociaux par des milliers d'internautes change la donne dans les pratiques des professionnels. Comment intégrer ces nouveaux médias à nos stratégies de communication ? Ci-proposée, une démarche d'intégration en dix étapes. Mots-clés : Appropriation, communication, médias réseaux sociaux, professionnels, stratégies d'intégration.

Abstract : Social networks appropriation by million of webusers changes the playing field in professionals' practices. How to integrate those new media into our communication strategies? Here's a proposed 10 step approach.

Keywords : Appropriation, Communication, Integrated practices, Professional, Social media network. 
